\title{
Numerical solution and spectrum of boundary-domain integral equation for the Neumann BVP with variable coefficient
}

\author{
Sergey E. Mikhailov* and Nurul A. Mohamed \\ Department of Mathematics, Brunel University London, Uxbridge, UB3 8PH, UK \\ (Received 00 Month 200x; in final form 00 Month 200x)
}

\begin{abstract}
A numerical implementation of a direct united Boundary-Domain Integral Equation (BDIE) related to the Neumann boundary value problem for a scalar elliptic PDE with variable coefficient is discussed. The BDIE is reduced to a uniquely solvable one by adding an appropriate perturbation operator. The mesh-based discretisation of the BDIEs with quadrilateral domain elements leads to a system of linear algebraic equations (discretised BDIE). Then the system is solved by LU decomposition and the Neumann iterations. Convergence of the iterative method is discussed in relation to the distribution of eigen-values of the corresponding discrete operators calculated numerically.
\end{abstract}

Keywords: boundary-domain integral equations, numerical solution, iterative methods, spectrum, eigen-values

AMS Subject Classification: Mathematics Subject Classification 2010: 65N38, 31A10, 45A05, 45C05, 65R20

\section{Introduction}

It is well known that one can reduce a boundary-value problem (BVP) for a linear partial differential equation (PDE) to a boundary-integral equation (BIE) and then solve the latter numerically. However, in order for the reduction to be enabled, a fundamental solution for the PDE is necessary. Even though fundamental solutions are known for many equations with constant coefficients, they are not generally available in an explicit form for partial differential operators with variable coefficients.

In handling the variable-coefficient cases, one can use a parametrix (Levi function), which is much wider available, instead of the fundamental solution. This approach allows reduction of the PDEs with variable coefficients not to BIE but to a boundary-domain integral equation (BDIE) or a boundary-domain integrodifferential equation (BDIDE), cf. [10, 11, 14].

Let us consider the Neumann problem for the following linear second-order elliptic PDE in a bounded domain $\Omega \subset \mathbb{R}^{2}$ with a boundary $\partial \Omega$ :

$$
\begin{aligned}
& A u(x):=\sum_{i=1}^{2} \frac{\partial}{\partial x_{i}}\left[a(x) \frac{\partial u(x)}{\partial x_{i}}\right]=f(x), \quad x \in \Omega, \\
& T u(x):=\sum_{j=1}^{2} a(x) n_{j}(x) \frac{\partial u(x)}{\partial x_{j}}=\bar{t}(x), \quad x \in \partial \Omega,
\end{aligned}
$$

\footnotetext{
*Corresponding author. Email: Sergey.Mikhailov@brunel.ac.uk
} 
where $u(x)$ is the unknown function, while $f(x), \bar{t}(x)$ and $a(x)>$ const $>0$ are prescribed functions. It is well established that the Neumann problem (1)-(2) is solvable if and only if the right hand sides in (1)-(2) satisfy the solvability (compatibility) condition

$$
\int_{\Omega} f(x) d x=\int_{\partial \Omega} \bar{t}(x) d \Gamma(x)
$$

When the condition is satisfied, the solution $u(x)$ does exist but is non-unique and the difference between any two solutions is a constant.

A parametrix (Levi function) for PDE (1) with variable coefficient, obtained from the fundamental solution for the same equation but with 'frozen' coefficient $a(x)=a(y)$, is

$$
P(x, y)=\frac{\ln |x-y|}{2 \pi a(y)}, \quad x, y \in \mathbb{R}^{2} .
$$

It satisfies equation

$$
A_{x} P(x, y)=\delta(x-y)+R(x, y)
$$

where $\delta(x-y)$ is the Dirac delta-function, while the remainder

$$
R(x, y)=\frac{1}{2 \pi a(y)} \sum_{i=1}^{2} \frac{x_{i}-y_{i}}{|y-x|^{2}} \frac{\partial a(x)}{\partial x_{i}}, \quad x, y \in \mathbb{R}^{2},
$$

has only a weak singularity at $x=y$, see [10].

The derivation of some BDIEs for the Dirichlet, Neumann and mixed problems for PDE (1) with variable coefficient can be found in [10]. Particularly, the direct united BDIE for the Neumann problem with respect to the unknown function $u$ has the following form (cf. also analysis of the united BDIEs for the mixed BVP in $[12])$ :

$$
c(y) u(y)-\int_{\partial \Omega} u(x) T_{x} P(x, y) \mathrm{d} \Gamma(x)+\int_{\Omega} R(x, y) u(x) \mathrm{d} x=\mathcal{F}(y), \quad y \in \Omega \cup \partial \Omega,
$$

where

$$
\mathcal{F}(y)=-\int_{\partial \Omega} P(x, y) \bar{t}(x) \mathrm{d} \Gamma(x)+\int_{\Omega} P(x, y) f(x) \mathrm{d} x .
$$

The first integral in (6) is understood in the Cauchy principal value sense if $y \in \partial \Omega$, and

$$
c(y)=\left\{\begin{array}{cl}
1 & \text { if } y \in \Omega, \\
0 & \text { if } y \in \mathbb{R}^{2} \backslash \bar{\Omega}, \\
\alpha(y) / 2 \pi & \text { if } y \in \partial \Omega
\end{array}\right.
$$

where $\bar{\Omega}=\Omega \cup \partial \Omega$ and $\alpha(y)$ is an interior angle at a corner point $y$ of the boundary $\partial \Omega$, if the corners are present. If $y$ belongs to a smooth part of the boundary, then $c(y)=1 / 2$.

BDIE (6) does not only contain the usual line integrals over the boundary $\partial \Omega$ as in the case when the parametrix is a fundamental solution but also integrals 
over the entire domain $\Omega$ with the unknown function $u$ in the integrand. BDIE (6) is called united since the unknown function $u$ on the boundary is just a trace of the function $u$ in the domain, in contrast to the so-called segregated BDIEs, where the unknown boundary functions are considered as formally independent of the unknown variables in the domain, cf. [2, 4, 10, 12].

In [14], an equation similar to (6) but with a localised parametrix instead of (4) was perturbed by a finite-dimensional operator and discretised using triangular linear domain elements and linear boundary elements. Then the obtained linear algebraic system was solved by the direct (LU decomposition) method.

The present paper develops some preliminary results published in [13]. After perturbation of (6) by a finite-dimensional operator to obtain an unconditionally solvable BDIE, we discretise it by quadrilateral bi-linear domain elements and linear boundary elements, and solve the resulting system of linear algebraic equations by a version of Neumann iterations and compare the solution with the results obtained by LU decomposition. To investigate the dependence of the convergence of the iterative method on the PDE coefficient, we calculated also the eigen-values of the resulting algebraic systems approximating the eigen-values of the BDIEs.

\section{Perturbation of the BDIE}

Let us introduce the operator $\tilde{\mathcal{K}}$,

$$
\begin{aligned}
{[\tilde{\mathcal{K}} u](y):=(1-c(y)) u(y)+\int_{\partial \Omega} u(x) T_{x} P(y, x) } & \mathrm{d} \Gamma(x) \\
& -\int_{\Omega} u(x) R(y, x) d x, \quad y \in \bar{\Omega} .
\end{aligned}
$$

Then BDIE (6) can be rewritten in a more compact form as

$$
u(y)-[\tilde{\mathcal{K}} u](y)=\mathcal{F}(y), \quad y \in \bar{\Omega}
$$

It was already mentioned that the Neumann problem (1)-(2) is not unconditionally solvable, and when it is solvable, its solution can only be unique up to an additive constant. These properties are inherited by BDIE (9), cf. [4]. Particularly, any constant solves the homogeneous equation (9), while the non-homogeneous equation (9) is solvable if and only if the the right hand side $\mathcal{F}(y)$ satisfies a solvability condition. One can show that if the BVP solvability condition (3) is satisfied, then the solvability condition for (9) will be satisfied as well. However, even in this case, a numerical solution of (9) would be unstable. To avoid this, similar to [14], one can add to equation (9) the perturbation operator

$$
[\stackrel{\circ}{\mathcal{K}} u](y):=\frac{1}{|\partial \Omega|} \int_{\partial \Omega} u(x) \mathrm{d} \Gamma(x), \quad y \in \bar{\Omega},
$$

where $|\partial \Omega|:=\int_{\partial \Omega} \mathrm{d} \Gamma(x)$ is the boundary length, and arrive at the following perturbed equation

$$
u(y)-[\hat{\mathcal{K}} u](y)=\mathcal{F}(y), \quad y \in \bar{\Omega},
$$


where

$$
\hat{\mathcal{K}}:=\tilde{\mathcal{K}}-\stackrel{\circ}{\mathcal{K}}
$$

is the perturbed boundary-domain integral operator. It is well known, cf. e.g. [5, $\S 609]$ ), that there is one eigen-function and no associated functions corresponding to the eigen-value $\tilde{\lambda}=1$ of the operator $\tilde{\mathcal{K}}$ for $a=$ const, i.e., both algebraic and geometric multiplicities are the same. We assume that there are no associated functions corresponding to the eigen-value $\tilde{\lambda}=1$ also for the case of the variable coefficient $a(x)$. Then following [9], one can prove that equation (11) is uniquely solvable for any right-hand side and moreover, when the solvability condition for equation (9) is satisfied, one of its solutions, such that $\int_{\partial \Omega} u(x) \mathrm{d} \Gamma(x)=0$, is delivered by the solution of its perturbed counterpart (11).

\section{Discretization of the BDIE}

The domain $\Omega$ is discretised by a mesh of $M$ iso-parametric quadrilateral bilinear domain elements, $\bar{\Omega}=\bigcup_{m}^{M} \bar{e}_{m}, e_{k} \cap e_{m}=\emptyset$ if $k \neq m$, with nodes $x^{i}, i=1, \ldots, J$, at the vertices of quadrilaterals. The Cartesian coordinates of a point on domain element $e_{m} \subset \Omega$ with the vertices $X^{m N}, N=1, \ldots, 4$, in terms of the intrinsic coordinates $\left(\xi_{1}, \xi_{2}\right)=: \xi$ on the reference square are given by the relations

$$
x(\xi)=\sum_{N=1}^{4} \Phi_{N}(\xi) X^{m N}, \quad-1 \leq \xi_{1} \leq 1,-1 \leq \xi_{2} \leq 1,
$$

where $\Phi_{N}(\xi)$ are the local shape functions,

$$
\begin{aligned}
& \Phi_{1}(\xi)=\left(1-\xi_{1}\right)\left(1-\xi_{2}\right) / 4, \Phi_{2}(\xi)=\left(1+\xi_{1}\right)\left(1-\xi_{2}\right) / 4 \\
& \Phi_{3}(\xi)=\left(1+\xi_{1}\right)\left(1+\xi_{2}\right) / 4, \Phi_{4}(\xi)=\left(1-\xi_{1}\right)\left(1+\xi_{2}\right) / 4
\end{aligned}
$$

Similar to the finite element approximation, the unknown function $u(x)$ at any point $x \in \bar{\Omega}$ is interpolated over its values $u\left(x^{j}\right)$ at the global nodes $x^{j}$ as,

$$
u(x)=\sum_{j} \phi_{j}(x) u\left(x^{j}\right), \quad x, x^{j} \in \bar{\Omega},
$$

where $\phi_{j}(x)$ are the global shape functions satisfying the so-called $\delta$-property, $\phi_{j}\left(x_{k}\right)=\delta_{j k}$, and related with the local shape functions as

$$
\phi_{j}(x)= \begin{cases}\Phi_{N}\left(\xi^{m}(x)\right) & \text { if } \quad x \in \bar{e}_{m}, x^{j}=X^{m N} \in\left\{X^{m N^{\prime}}\right\}_{N^{\prime}=1}^{4} \\ 0 & \text { if } \quad x \in \bar{e}_{m}, x^{j} \notin\left\{X^{m N^{\prime}}\right\}_{N^{\prime}=1}^{4}\end{cases}
$$

where $\xi^{m}(x)$ are the functions inverse to (12).

The polygonal boundary $\partial \Omega$ becomes discretised with $L$ continuous linear isoparametric elements, $\partial \Omega=\bigcup_{l}^{L} \Gamma_{l}$, where $\Gamma_{1}, \Gamma_{2}, \cdots, \Gamma_{L}$ are the sides of the corresponding domain elements. The Cartesian coordinates of a point on a boundary element $\Gamma_{l} \subset \partial \Omega$ with the intrinsic coordinate $\eta$, which coincides with an intrinsic 
coordinate $\xi_{1}$ or $\xi_{2}$ of the corresponding domain element, are given by

$$
x(\eta)=\sum_{n=1}^{2} \Psi_{n}(\eta) X^{l n}, \quad-1 \leq \eta \leq 1,
$$

where $\Psi_{n}(\eta)$ are the local one-dimensional shape functions, that are the traces of the two-dimensional shape functions $\Phi_{N}(\xi)$ :

$$
\Psi_{1}(\eta)=\frac{1}{2}(1-\eta), \quad \Psi_{2}(\eta)=\frac{1}{2}(1+\eta), \quad-1 \leq \eta \leq 1 .
$$

Applying the interpolation to equation (11) employed at the mesh nodes $x^{i}$, $i=1, \ldots, J$, as the collocation points, we get the system of $J$ linear algebraic equations for $J$ unknowns $u\left(x^{j}\right)$,

$$
u\left(x^{i}\right)-\sum_{x^{j} \in \bar{\Omega}} \tilde{K}_{i j} u\left(x^{j}\right)+\sum_{x^{j} \in \partial \Omega} \stackrel{\circ}{K}_{i j} u\left(x^{j}\right)=F_{i}, \quad x^{i} \in \bar{\Omega}, \quad i=1, \cdots, J,
$$

where $F_{i}=Q_{i}+D_{i}$,

$$
\begin{aligned}
& \tilde{K}_{i j}:= {\left[\tilde{\mathcal{K}} \phi_{j}\right]\left(x^{i}\right) } \\
&=\left(1-c\left(x^{i}\right)\right) \delta_{i j}+\int_{\partial \Omega} \phi_{j}(x) T_{x} P\left(x, x^{i}\right) \mathrm{d} \Gamma(x)-\int_{\Omega} \phi_{j}(x) R\left(x, x^{i}\right) \mathrm{d} x \\
&=\left(1-c\left(x^{i}\right)\right) \delta_{i j}+\sum_{l=1}^{L} \int_{\Gamma_{l}} \phi_{j}(x) T_{x} P\left(x, x^{i}\right) \mathrm{d} \Gamma(x)-\sum_{m=1}^{M} \int_{e_{m}} \phi_{j}(x) R\left(x, x^{i}\right) \mathrm{d} x \\
&=\left(1-c\left(x^{i}\right)\right) \delta_{i j}+\sum_{\bar{\Gamma}_{l} \ni x^{j}} \int_{\Gamma_{l}} \phi_{j}(x) T_{x} P\left(x, x^{i}\right) \mathrm{d} \Gamma(x)-\sum_{\bar{e}_{m} \ni x^{j}} \int_{e_{m}} \phi_{j}(x) R\left(x, x^{i}\right) \mathrm{d} x \\
& \stackrel{\circ}{K_{i j}}:=\mathcal{K} \phi_{j}=\frac{1}{|\partial \Omega|} \int_{\partial \Omega} \phi_{j}(x) \mathrm{d} \Gamma(x) \frac{1}{|\partial \Omega|} \sum_{l=1}^{L} \int_{\Gamma_{l}} \phi_{j}(x) \mathrm{d} \Gamma(x)=\frac{1}{|\partial \Omega|} \sum_{\bar{\Gamma}_{l} \ni x^{j}} \int_{\Gamma_{l}} \phi_{j}(x) \mathrm{d} \Gamma(x) \\
& Q_{i}=-\int_{\partial \Omega} P\left(x, x^{i}\right) \bar{t}(x)=-\sum_{l=1}^{L} \int_{\Gamma_{l}} P\left(x, x^{i}\right) \bar{t}(x) \mathrm{d} \Gamma(x) \\
& P\left(x, x^{i}\right) f(x) \mathrm{d} x=\sum_{m=1}^{M} \int_{e_{m}} P\left(x, x^{i}\right) f(x) \mathrm{d} x \\
& D_{i}
\end{aligned}
$$

and $\delta_{i j}$ is the Kronecker symbol. Note that from (7) we have in first term of (15), $c\left(x^{i}\right)-1=0$ for the interior points $x^{i}$ and $c\left(x^{i}\right)-1=-1 / 2$ for the smooth points $x^{i}$ of the boundary $\partial \Omega$. It follows from (16) that the entries $\stackrel{\circ}{K}_{i j}$ do not in fact depend on $i$, and $\stackrel{\circ}{K}_{i j}=0$ if $x^{j} \notin \partial \Omega$. 
Denoting $\hat{K}_{i j}:=\tilde{K}_{i j}-\stackrel{\circ}{K}_{i j}$, algebraic system (14) can be presented also as

$$
u\left(x^{i}\right)-\sum_{x^{j} \in \bar{\Omega}} \hat{K}_{i j} u\left(x^{j}\right)=F_{i}, \quad x^{i} \in \bar{\Omega}, \quad i=1, \cdots, J
$$

After changing the integration variables to the intrinsic coordinates, we can write (15)-(18) as

$$
\begin{gathered}
\tilde{K}_{i j}=\left(1-c\left(x^{i}\right)\right) \delta_{i j}+\sum_{\bar{\Gamma}_{l} \ni x^{j}} A_{n(j, l), i}^{l}-\sum_{\bar{e}_{m} \ni x^{j}} G_{N(j, m), i}^{m}, \\
\stackrel{\circ}{K}_{i j}=\frac{1}{|\partial \Omega|} \sum_{\bar{\Gamma}_{l} \ni x^{j}} B_{n(j, l)}^{l}, \quad Q_{i}=-\sum_{l=1}^{L} C_{i}^{l}, \quad D_{i}=\sum_{m=1}^{M} H_{i}^{m},
\end{gathered}
$$

where $n(j, l)$ is the local number of the node $x^{j}$ on the boundary element $\Gamma_{l}$, $N(j, m)$ is the local number of the node $x^{j}$ on the domain element $e_{m}$,

$$
\begin{aligned}
A_{n i}^{l} & =\int_{-1}^{1} \Psi_{n}(\eta) T_{x} P\left(x(\eta), x^{i}\right) J_{l 1}(\eta) \mathrm{d} \eta, \\
B_{n}^{l} & =\int_{-1}^{1} \Psi_{n}(\eta) J_{l 1}(\eta) \mathrm{d} \eta, \\
C_{i}^{l} & =\int_{-1}^{1} P\left(x(\eta), x^{i}\right) \bar{t}(x(\eta)) J_{l 1}(\eta) \mathrm{d} \eta, \\
G_{N i}^{m} & =\int_{-1}^{1} \int_{-1}^{1} \Phi_{N}(\xi) R\left(x(\xi), x^{i}\right) J_{m 2}(\xi) \mathrm{d} \xi_{1} \mathrm{~d} \xi_{2}, \\
H_{i}^{m} & =\int_{-1}^{1} \int_{-1}^{1}\left(P\left(x(\xi), x^{i}\right) f(x(\xi)) J_{m 2}(\xi) \mathrm{d} \xi_{1} \mathrm{~d} \xi_{2},\right.
\end{aligned}
$$

and $J_{m 2}$ and $J_{l 1}$ are the Jacobians of the transforms (12) and (13), respectively.

The regular integral (21) and the double layer potential (20) (since it is regular on the piece-wise smooth curves) as well as the integrals in (22)-(24), when the collocation point $x^{i}$ is not a vertex of the integration element, are evaluated by the Gauss-Legendre quadrature formulas,

$$
\int_{-1}^{1} f(\eta) \mathrm{d} \eta=\sum_{p=1}^{\imath} W_{p} f\left(\eta_{p}\right), \quad \int_{-1}^{1} \int_{-1}^{1} f(\xi) \mathrm{d} \xi_{1} \mathrm{~d} \xi_{2}=\sum_{q=1}^{\jmath} \sum_{p=1}^{\imath} W_{p} W_{q} f\left(\xi_{1 p}, \xi_{2 q}\right),
$$

where $\imath$ and $\jmath$ are the numbers of quadrature points used to evaluate the integrals, $\eta_{p}, \xi_{1 p}$ and $\xi_{2 q}$ are the quadrature point coordinates, while $W_{p}$ and $W_{q}$ are the quadrature weights associated with points $p$ and $q$, respectively.

However, the integrals (22)-(24) need a special treatment when a collocation point $x^{i}$ is a vertex of the integration element since the kernels of these integrals are weakly singular at collocation points. The integrals (22) with the kernel involving $\ln (1 / r)$ are evaluated by the Gauss-Laguerre quadrature, i.e.,

$$
\int_{0}^{1} f(\bar{\eta}) \ln \left(\frac{1}{\bar{\eta}}\right) \mathrm{d} \eta \approx \sum_{p=1}^{\imath} W_{p} f\left(\bar{\eta}_{p}\right)
$$


cf. [1]. For the domain integrals (23) and (24), we split the square reference element into triangular sub-elements and apply the Duffy transformation, cf. [1].

System (19) can now be solved by a numerical method for linear algebraic systems, particularly LU decomposition method or the Neumann series expansion

$$
\mathbf{u}=\sum_{n=0}^{\infty} \hat{\mathbf{K}}^{n} \mathbf{F},
$$

where $\hat{\mathbf{K}}=\left\{\hat{K}_{i j}\right\}_{i, j=1}^{J}, \mathbf{u}=\left\{u\left(x^{j}\right)\right\}_{j=1}^{J}, \mathbf{F}=\left\{F\left(x^{i}\right)\right\}_{j=1}^{J}$.

Convergence of the Neumann series of the form (25) for the purely boundary integral operator associated with the Dirichlet problem for the Laplace equation is well known, see e.g. $[5,15,16]$. For the perturbed boundary integral operator associated with the Neumann problem for the Laplace equation the convergence is shown, for example, in [8]. To the best of the authors knowledge, a proof of convergence of the Neumann series for BDIEs is not available. One of the objectives of the paper is to conclude from numerical experiments whether series (25) does converge, for discretised BDIE (19), in the considered examples.

\section{Numerical solution of BDIEs}

A FORTRAN code was written for numerical solution of the BDIEs where the system of equation (19) is solved by the LU decomposition method and by the Neumann series expansion (25). Let us define the relative errors for the approximate solution and for its gradient as

$$
\begin{aligned}
\varepsilon(u) & =\max _{1 \leq j \leq J}\left|u_{\text {approx }}\left(x^{j}\right)-u_{\text {exact }}\left(x^{j}\right)\right| / \max _{1 \leq j \leq J}\left|u_{\text {exact }}\left(x^{j}\right)\right|, \\
\varepsilon(\nabla u) & =\max _{1 \leq m \leq M}\left|\nabla u_{\text {approx }}\left(x_{c}^{m}\right)-\nabla u_{\text {exact }}\left(x_{c}^{m}\right)\right| / \max _{1 \leq m \leq M}\left|\nabla u_{\text {exact }}\left(x_{c}^{m}\right)\right|,
\end{aligned}
$$

where $x_{c}^{m}$ are centres of the quadrilateral domain elements $e_{m}$. We will analyse in this section convergence for the square, circular and parallelogram domains (see Figures 1, 4 and 7, respectively) and present graphs of these errors versus the number of the mesh nodes $J$ for the algebraic system solution by the LU decomposition and also versus the number of iterations for the solution by the Neumann iteration method.

For each domain we solve the following two test interior Neumann problems (1)-(2) with $a(x)=x_{2}^{2}$.

Test 1: $f(x)=0$ in $\Omega, \bar{t}(x)=x_{2}^{2} n_{1}(x)$ on $\partial \Omega$, with $u_{\text {exact }}(x)=x_{1}$ in $\bar{\Omega}$.

Test 2: $f(x)=2 x_{2}^{2}$ in $\Omega, \bar{t}(x)=2 x_{1} x_{2}^{2} n_{1}(x)$ on $\partial \Omega$, with $u_{\text {exact }}(x)=x_{1}^{2}$ in $\bar{\Omega}$.

Figures 2(a), 5(a), 8(a) give the dependence of the solution error on the number of collocation points $J$ for the solution by the LU decomposition and by the Neumann iterations. The dependence of the error $\varepsilon(u)$ on the number of nodes $J$ (an on the average linear size of the elements, $h$ ) can be fitted with a power function (i.e. with a straight line in the double logarithmic coordinates in the graphs), giving $\varepsilon(u) \sim J^{-q / 2} \sim h^{q}$, where $q \approx 1$ in Test 1 and $q \approx 2$ in Test 2, i.e., respectively, linear and quadratic convergence with respect to the element size $h$. For the gradient error we similarly have $\varepsilon(\nabla u) \sim J^{-q^{\prime} / 2} \sim h^{q^{\prime}}$, where $q^{\prime} \approx 0.1$ for the square and circular domains and $q^{\prime} \approx 0.2$ for the parallelogram domain in Test 1 , while $q^{\prime} \approx 1$ for the square and parallelogram domains and $q^{\prime} \approx 2$ for the circular domain in Test 2 . 


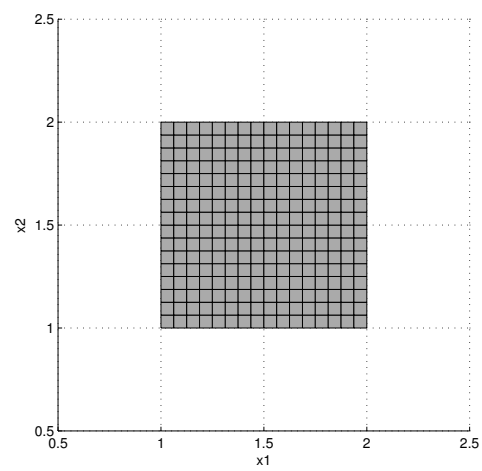

Figure 1. The square domain $1<x_{1}<2,1<x_{2}<2$.

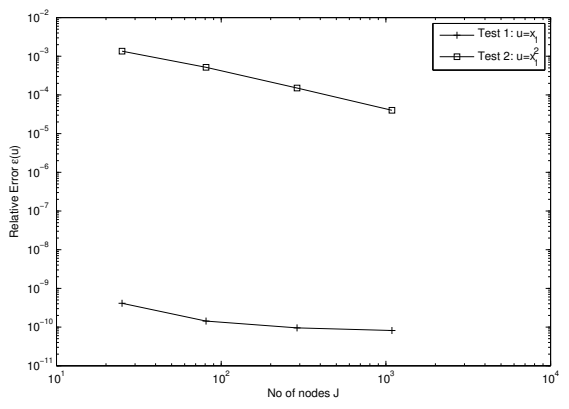

(a)

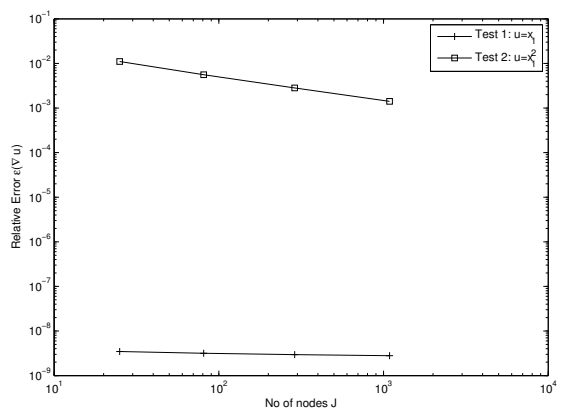

(b)

Figure 2. Relative error of the approximate solutions (a) and their gradients (b), on the square vs. number of nodes $J$.

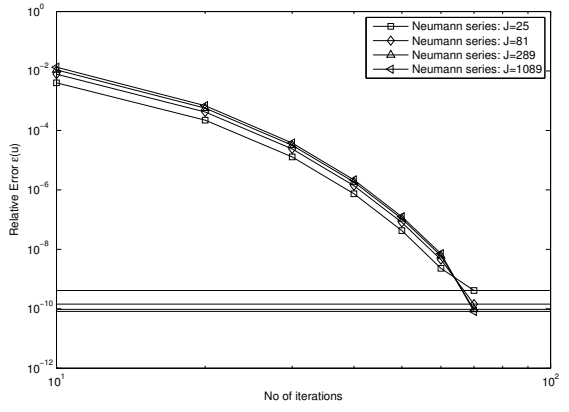

(a) Test 1

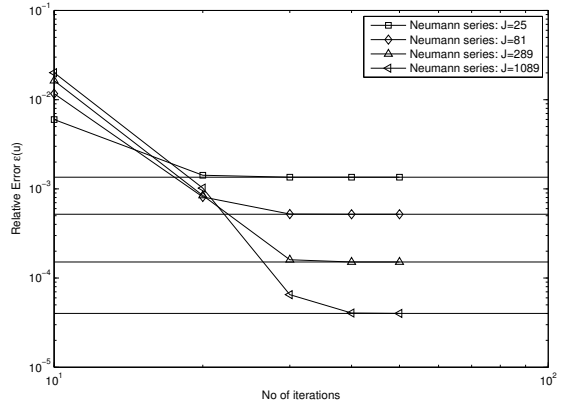

(b) Test 2

Figure 3. Relative error of the solutions on the square vs. number of Neumann iterations, compared with the error of the LU decomposition solution (horizontal lines), for different number of mesh nodes $J$.

The accuracy in Test 1 is much higher since the implemented piece-wise bi-linear interpolation is exact on the linear exact solution, and only the integral operator approximation error, related with the accuracy of the numerical integration, is involved. In the Test 2, on the contrary, the piece-wise bi-linear interpolation of the quadratic exact solution gives the major contribution to the total error. On the other hand, the higher convergence rate in Test 2 can be attributed to the quadratic convergence rate of the piece-wise linear interpolation of smooth nonlinear functions, while the lower convergence rate in Test 1 can be explained by the lower convergence rate of the approximation of the integral operator.

From Figs. 3, 6, 9 it can be seen that the Neumann series converge to the LU 


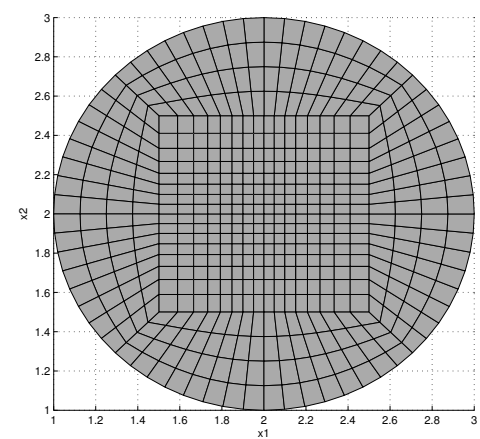

Figure 4. The unit-radius circular domain centred at $(2,2)$.

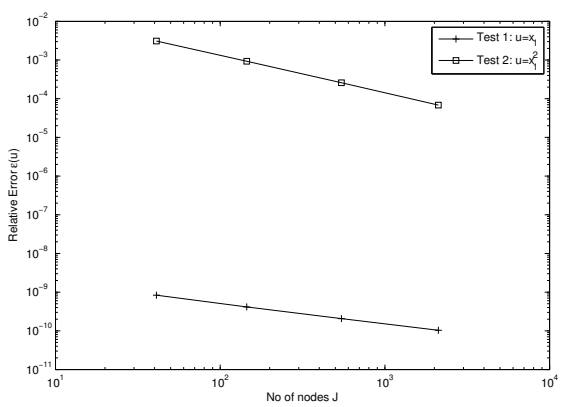

(a)

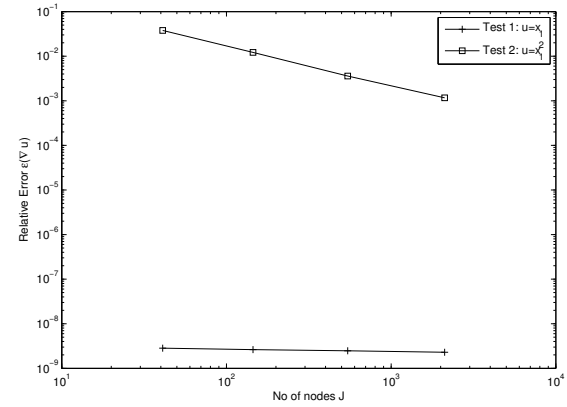

(b)

Figure 5. Relative error of the approximate solutions (a) and their gradients (b), on the circle vs. number of nodes $J$.

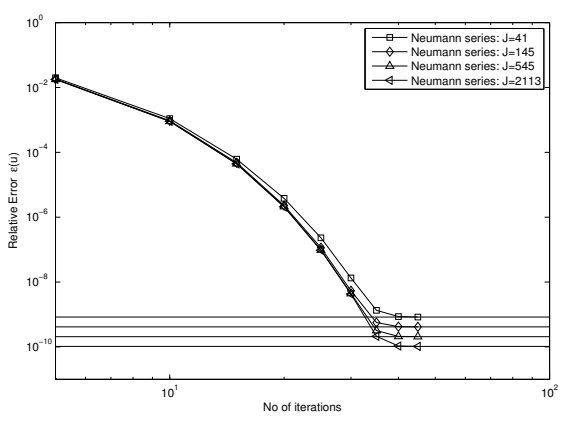

(a) Test 1

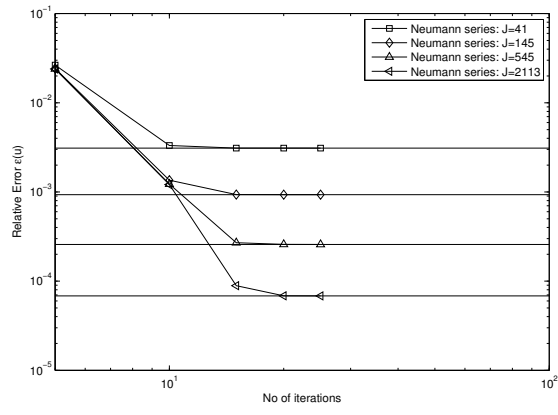

(b) Test 2

Figure 6. Relative error of the solutions on the circle domain vs. number of Neumann iterations, compared with the error of the LU decomposition solution (horizontal lines), for different number of mesh nodes $J$.

decomposition solutions, reaching the LU decomposition accuracy after 70 iterations for the square, 40 iterations for the circle and 140-160 iterations for the parallelogram in Test 1 and after 20-40 iterations for the square, 15-20 iterations for the circle and 60-100 iterations for the parallelogram in Test 2. The number of Neumann iterations necessary to reach the same accuracy as the LU decomposition grows slightly with the the number of collocation points since the accuracy of the LU decomposition numerical solution, taken for comparison, grows as well. The dependence of the iteration number on the test (i.e. on the exact solution behaviour) and on the domain shape is also related to the different accuracy of the LU numerical solution taken for comparison. 


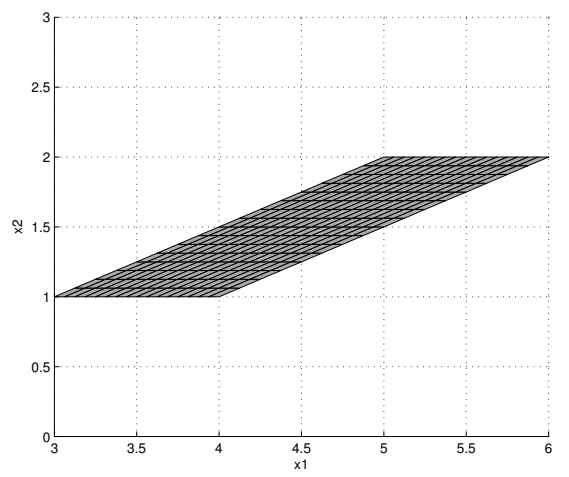

Figure 7. The parallelogram domain with vertices $(3,1),(4,1),(6,2),(5,2)$

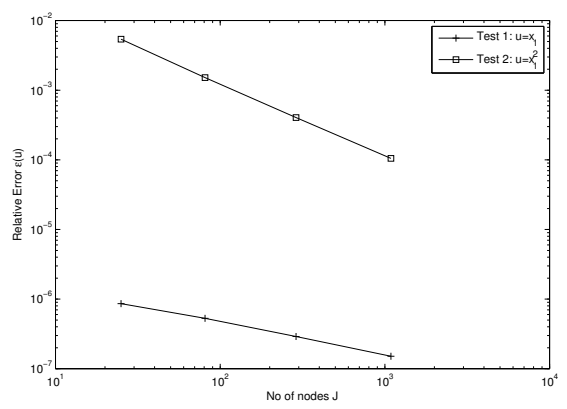

(a)

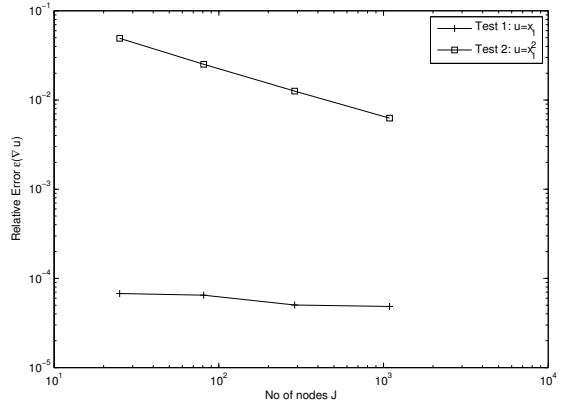

(b)

Figure 8. (a) The parallelogram domain with vertices $(3,1),(4,1),(6,2),(5,2)$, with $J=289$ node mesh. (b) Relative error of the approximate solutions (a) and their gradients (b), on the parallelogram vs. number of nodes $J$.

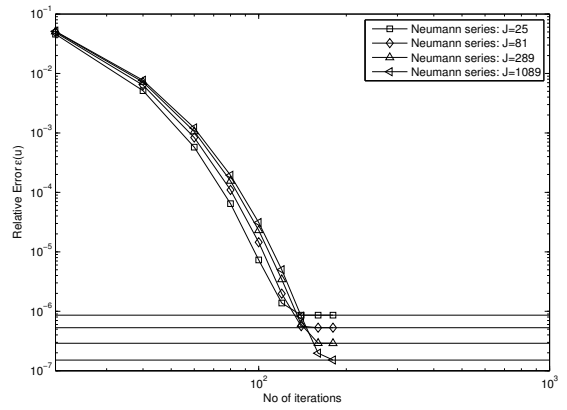

(a) Test 1

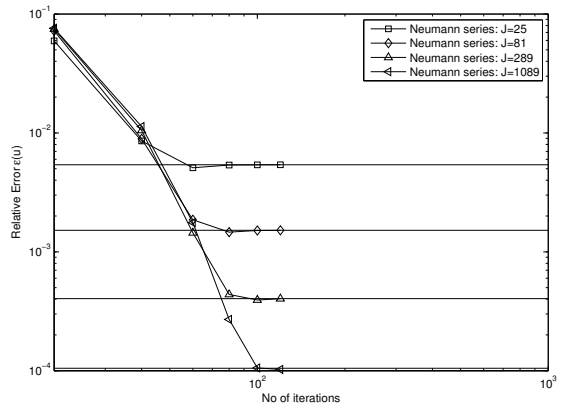

(b) Test 2

Figure 9. Relative error of the solutions on the parallelogram domain vs. number of Neumann iterations, compared with the error of the LU decomposition solution (horizontal lines), for different number of mesh nodes $J$. 


\section{Eigen-values}

To analyse the fast convergence of the iterative method in the examples considered in Section 4 and investigate whether it holds for other variable coefficients of the $\mathrm{PDE}$, we consider in this section the eigen-values of the obtained algebraic systems approximating the BDIEs. It is well known that the Neumann series in the form (25) for a matrix operator $\hat{\mathbf{K}}$ converges for any right hand side if and only if all eigen-values of the operator $\hat{\mathbf{K}}$ belong to the open unit disc. Moreover, the number of terms in the Neumann series sufficient for the error to be lower than a prescribed value, can be estimated in terms of the maximum eigen-value modulus.

Let $\tilde{\lambda}_{k}, k=1,2, \ldots, J$, denote the eigen-values of the matrix $\tilde{\mathbf{K}}=\left\{\tilde{K}_{i j}\right\}_{i, j=1}^{J}$, i.e., the numbers $\tilde{\lambda}_{k}$ for which the homogeneous equation

$$
\left(\tilde{\lambda}_{k} \mathbf{I}-\tilde{\mathbf{K}}\right) \mathbf{u}=0
$$

has non-trivial solutions. Similarly, let $\hat{\lambda}_{k}, k=1,2, \ldots, J$, denote the eigen-values of the perturbed matrix $\hat{\mathbf{K}}$.

When the coefficient $a(x)$ is constant, the remainder $R$ vanishes and boundarydomain integral equation (9) can be split into the purely boundary integral equation for the boundary values (traces) of $u$ on $\partial \Omega$, and in the representation formula for $u$ in $\Omega$. The same will hold also for the perturbed equation (11) and its discrete counterpart (19). From [5, 15] one can deduce that in this case the eigen-values of the non-perturbed boundary integral operator (and thus the whole operator $\tilde{\mathcal{K}})$ in the appropriate function spaces are real and belong to the segment $[0,1]$. Application of [9] gives that the spectrum of the perturbed operator $\hat{\mathcal{K}}$ belongs to the interval $[0,1)$, that is, its spectral norm is less than 1 implying convergence of the corresponding Neumann series.

When the coefficient $a(x)$ is not constant, the spectral properties and thus a proof of convergence of the Neumann series for BDIEs is not available, but some conclusions about the convergence can be drown from the following graphs presenting the numerically obtained largest-modulus eigen-values of the discrete operators $\tilde{\mathbf{K}}$ and $\hat{\mathbf{K}}$ and the influence of the coefficient $a$ on them.

Figures 10-12 show the first five eigen-values $\tilde{\lambda}_{k}$ of the matrix $\tilde{\mathbf{K}}$ with the largest moduli for the examples from Section 4 . These five eigen-values appear to be real for the square and parallelogram and have an imaginary part less than 0.006 for the circle. Numerically obtained largest eigen-values $\hat{\lambda}_{k}$ of the perturbed matrix $\hat{\mathbf{K}}$ coincide (up to the third digit) with those for the unperturbed matrix $\tilde{\mathbf{K}}$, except the eigen-value $\lambda_{1}=1$, that vanishes for $\hat{\mathbf{K}}$, as predicted by the theory. Indeed, the eigen-values of the discrete operators $\tilde{\mathbf{K}}$ and $\hat{\mathbf{K}}$ approximate the spectra of the corresponding integral operators $\tilde{\mathcal{K}}$ and $\hat{\mathcal{K}}$. The operators $\tilde{\mathcal{K}}$ and $\hat{\mathcal{K}}$ differ only by the perturbation operator (10) and, according to [9], their eigen-values coincide except the eigen-value $\tilde{\lambda}=1$ that is transferred to the spectrum point $\hat{\lambda}=0$, for the operator $\hat{\mathcal{K}}$, under the assumption that there are no associated functions corresponding to the eigen-value $\tilde{\lambda}=1$.

The maximal eigen-values of the matrix $\hat{\mathcal{K}}$, i.e., $\lambda_{2}$ on Figs. $10-12$ gives the spectral radius of the matrix $\hat{\mathcal{K}}$ influencing the convergence rate of the Neumann series. In our examples the radii are less than one, implying convergence of the Neumann series. For the circular domain it converges after 25 iterations, while for the parallelogram only after 100 iterations correlating well with $\max \left|\hat{\lambda}_{k}\right| \sim 0.5$ for the circular domain and $\max \left|\hat{\lambda}_{k}\right| \sim 0.9$ for the parallelogram.

To investigate the influence of the coefficient $a(x)$ on the maximum eigen-values of the perturbed matrix $\hat{\mathcal{K}}$, we calculated them for $a(x)=x_{2}^{k}$ with different $k \geq 1$. 


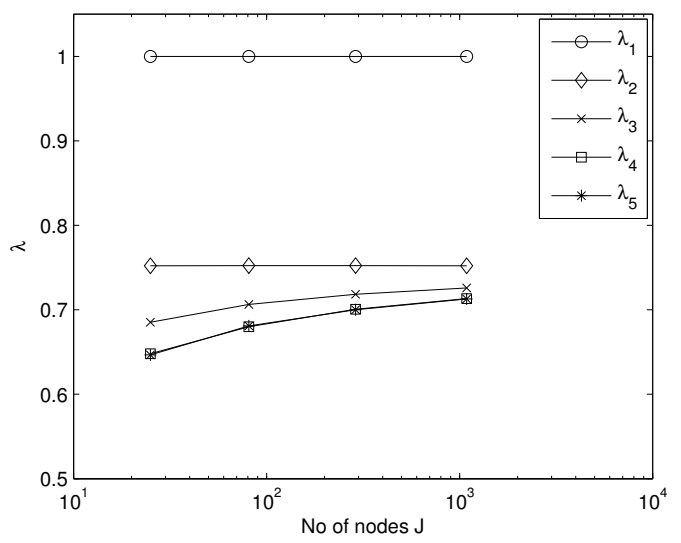

Figure 10. Eigen-values of the matrix $\tilde{\mathbf{K}}$ for the square vs. the number of nodes.

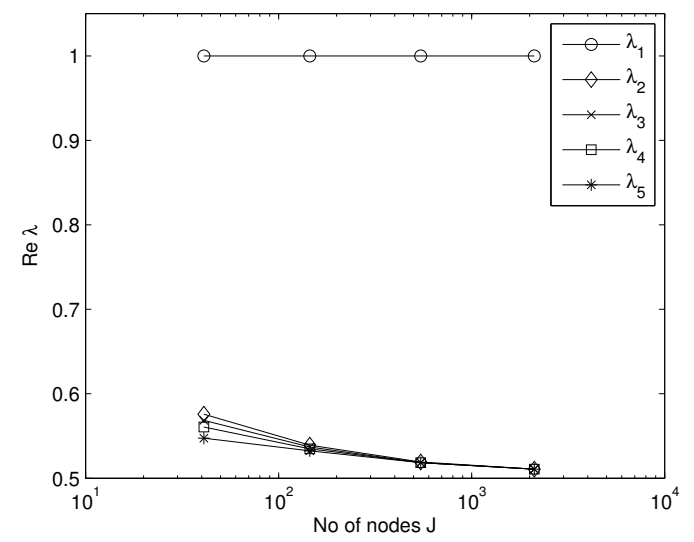

Figure 11. Eigen-values of the matrix $\tilde{\mathbf{K}}$ for the circular domain vs. the number of nodes.

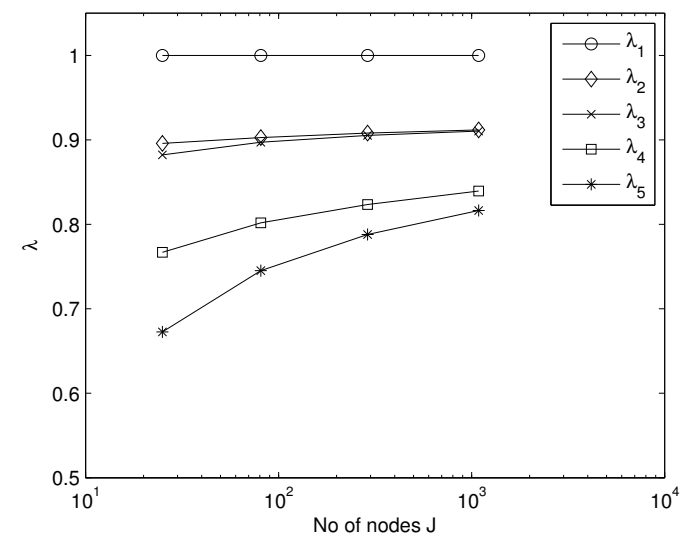

Figure 12. Eigen-values of the matrix $\tilde{\mathbf{K}}$ for the parallelogram vs. the number of nodes.

(Note that our previous examples were calculated for $k=2$.) The results are presented in Figs. 13-21 for the fine meshes, $J=1089$ for the square and parallelogram, and $J=2113$ for the circular domain. For the overlapping eigen-values seen on the figures our calculation shown that their eigen-functions are linearly independent, i.e., the eigen-values are geometrically multiple.

The figures show that for sufficiently high $k$, i.e., for sufficiently sharp variation of the coefficient, the eigen-values are generally complex and can lay outside the unit 
circle, unlike the constant-coefficient case. This means that the standard Neumann series for the BDIE with such variable coefficients can generally diverge. Note however that from these figures one can conclude that $0 \leq \operatorname{Re} \hat{\lambda}_{k}<1$ for the considered examples, similar to the constant coefficient case, while $\left|\operatorname{Im} \lambda_{k}\right|<C$ with some constant $C<1.5$. Following [6, Section 2.2.2], [7] one can map the exterior of this $\lambda$-domain to the exterior of the unit circle, which will lead to a converging modification of the Neumann series.

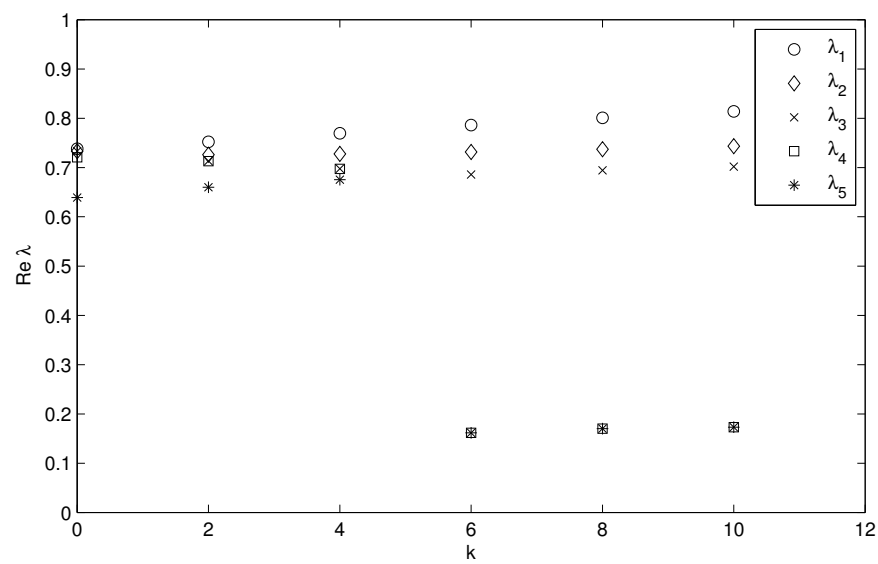

Figure 13. Real parts of the largest eigen-values of the matrix $\hat{\mathbf{K}}$ for the square vs. $k$ for $a(x)=x_{2}^{k}$.

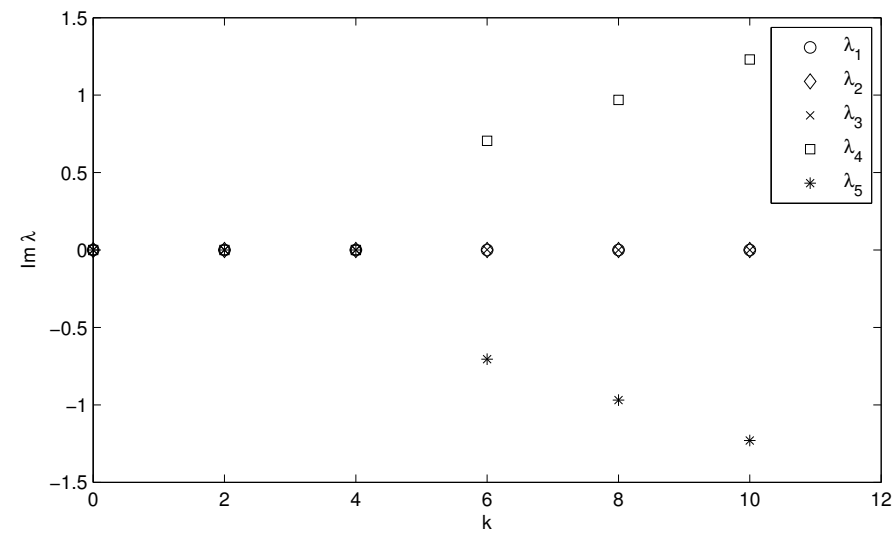

Figure 14. Imaginary parts of the largest eigen-values of the matrix $\hat{\mathbf{K}}$ for the square vs. $k$ for $a(x)=x_{2}^{k}$. 


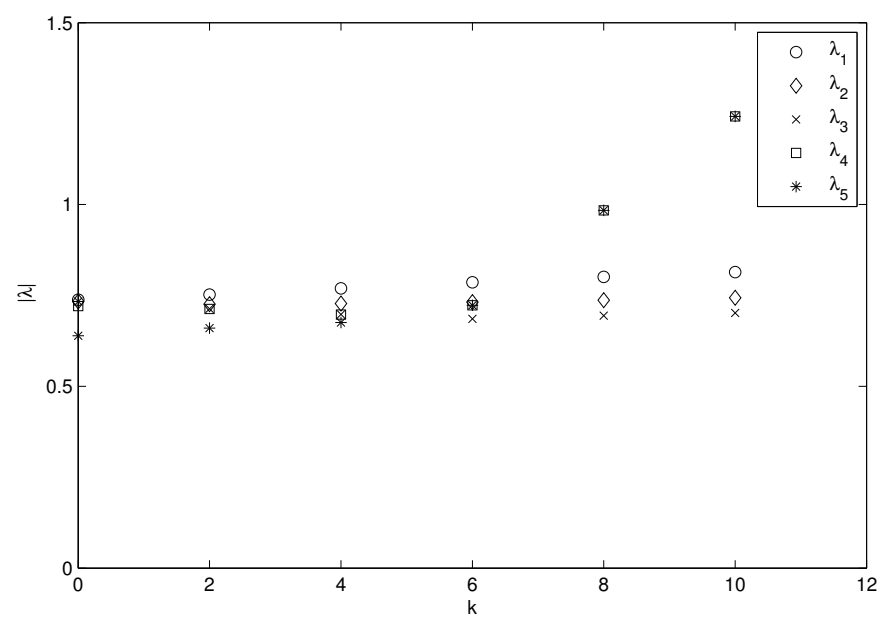

Figure 15. Moduli of the largest eigen-values of the matrix $\hat{\mathbf{K}}$ for the square vs. $k$ for $a(x)=x_{2}^{k}$.

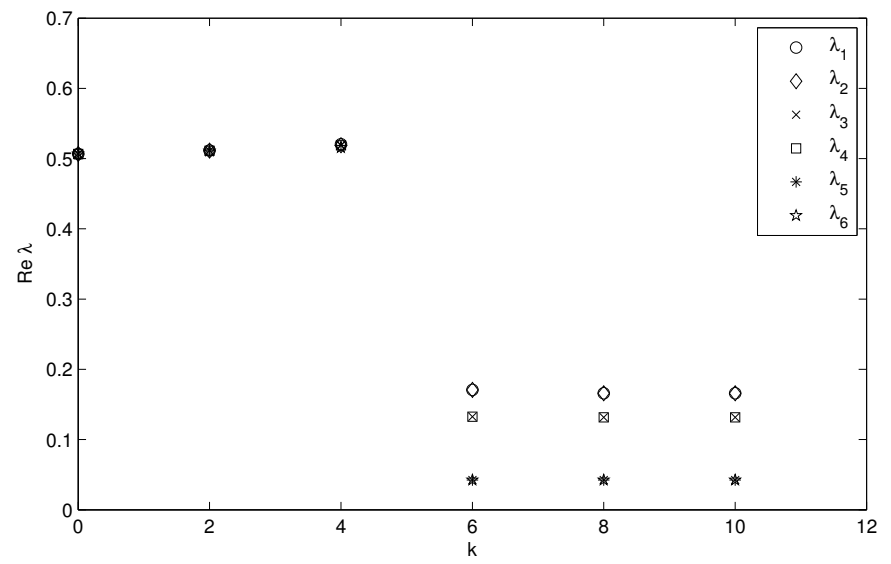

Figure 16. Real parts of the largest eigen-values of the matrix $\hat{\mathbf{K}}$ for the circular domain vs. $k$ for $a(x)=x_{2}^{k}$.

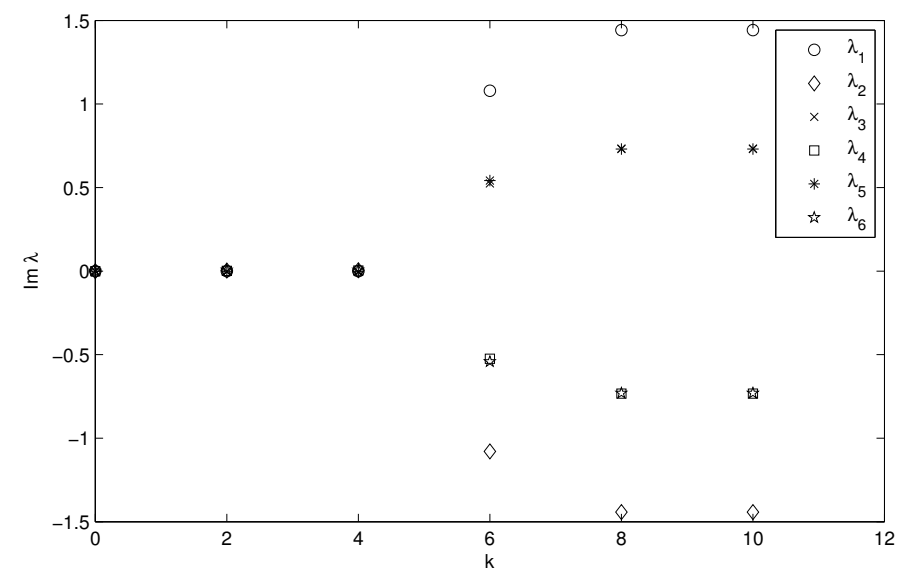

Figure 17. Imaginary parts of the largest eigen-values of the matrix $\hat{\mathbf{K}}$ for the circular domain vs. $k$ for $a(x)=x_{2}^{k}$. 


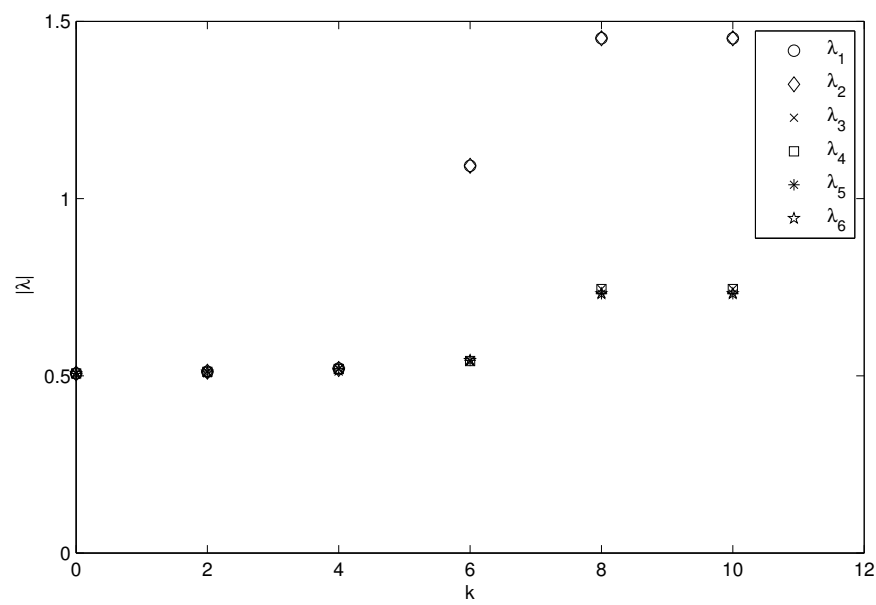

Figure 18. Moduli of the largest eigen-values of the matrix $\hat{\mathbf{K}}$ for the circular domain vs. $k$ for $a(x)=x_{2}^{k}$.

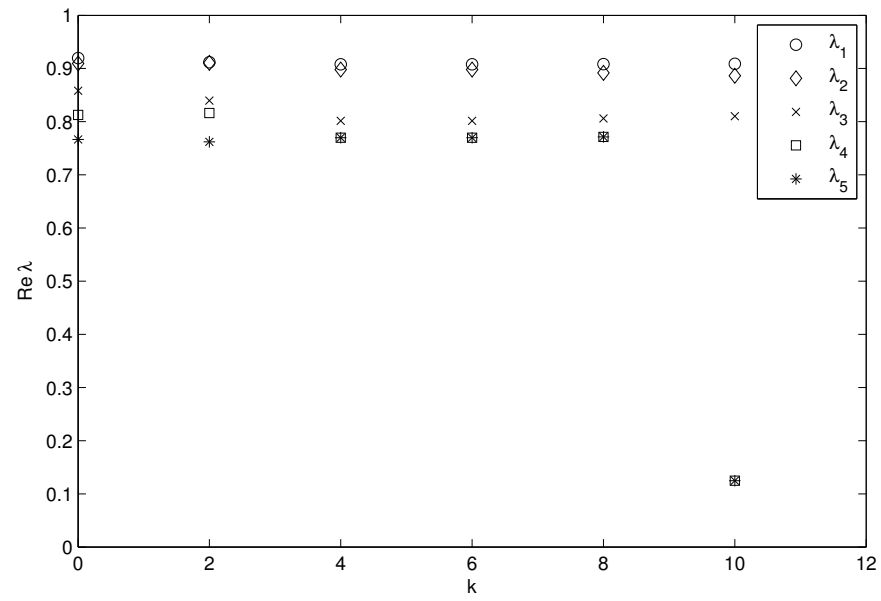

Figure 19. Real parts of the largest eigen-values of the matrix $\hat{\mathbf{K}}$ for the parallelogram vs. $k$ for $a(x)=x_{2}^{k}$.

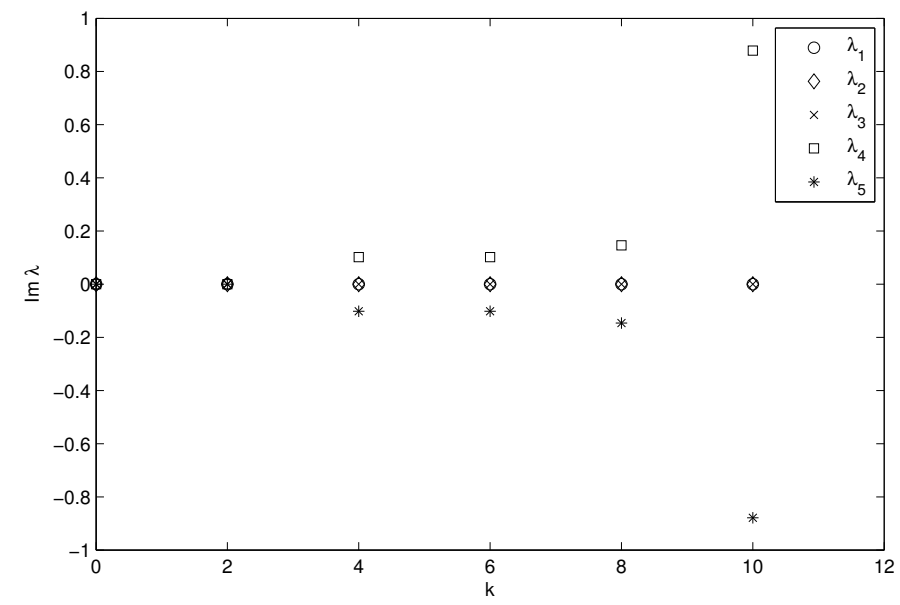

Figure 20. Imaginary parts of the largest eigen-values of the matrix $\hat{\mathbf{K}}$ for the parallelogram vs. $k$ for $a(x)=x_{2}^{k}$. 


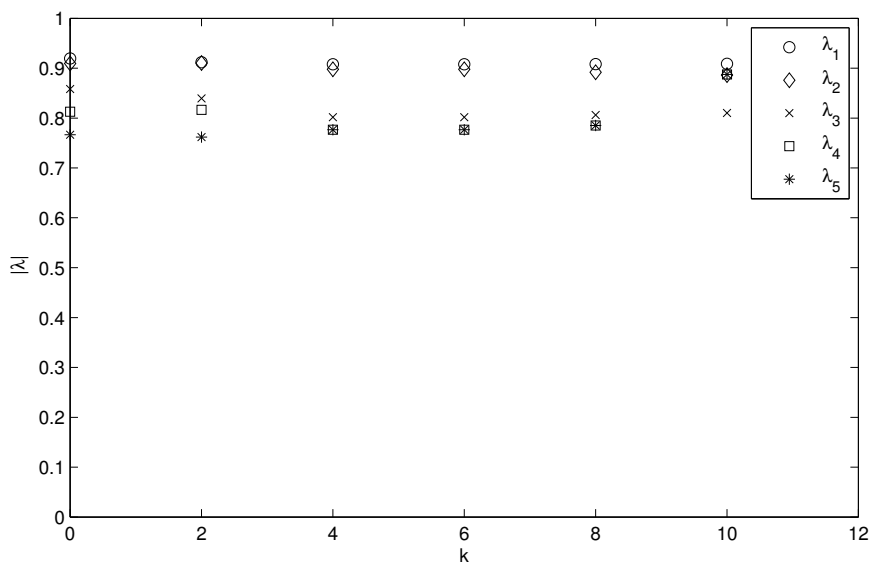

Figure 21. Moduli of the largest eigen-values of the matrix $\hat{\mathbf{K}}$ for the parallelogram vs. $k$ for $a(x)=x_{2}^{k}$.

\section{Concluding remarks}

The finite-dimensional perturbation allows to reduce the BDIE of the Neumann problem to an unconditionally and uniquely solvable integral equation.

The numerical results presented in the paper show that the mesh-based discretization of the BDIE with a quadrilateral bilinear approximation leads to a system of linear algebraic equations that can be solved e.g. by LU-decomposition with linear convergence with respect to the linear element size. For some variable coefficients and shapes of the domains, the discrete BDIE can be also solved by fast converging Neumann iterations, which is related to the beneficial spectral properties of the BDIE.

A more detailed analysis of the discrete BDIE eigen-values demonstrated that when the PDE coefficient moderately varies with coordinates, i.e., when the coefficient gradient is small or moderate (e.g. $|\nabla a|<5 a / L$ in the considered examples, where $L$ is a characteristic size of the domain) the spectrum is contained in the unit circle, which implies the Neumann series convergence. Then the standard Neumann iteration method is a good alternative to the direct methods, especially when the computer storage and CPU time needed for the latter become prohibitive. However, this spectrum property does not hold generally, and when the coefficient varies sharply enough, some eigen-values appear also outside the unit circle, which can lead to divergence of the standard Neumann series; in these cases the modified Neumann series, other iterative (e.g. GMRES) or direct methods will be more appropriate.

Further theoretical and numerical research is needed to analyse spectral properties of the localised BDIEs considered in $[3,11,14]$.

\section{Acknowledgment}

The work was supported by the grant EP/H020497/1 "Mathematical analysis of localised boundary-domain integral equations for BVPs with variable coefficients" of the EPSRC, UK. 


\section{References}

[1] G. Beer, Programming the Boundary Element Method, John Wiley \& Sons, West Sussex (2001).

[2] O. Chkadua, S.E. Mikhailov, and D. Natroshvili, Analysis of direct boundary-domain integral equations for a mixed BVP with variable coefficient, I: Equivalence and invertibility, Journal of Integral Equations and Applications 21 (2009), pp. 499-543.

[3] - Analysis of some localized boundary-domain integral equations, Journal of Integral Equations and Applications 21 (2009), pp. 405-445.

[4] - Analysis of segregated boundary-domain integral equations for variable-coefficient problems with cracks, Numerical Methods for Partial Differential Equations 27 (2011), pp. 121-140.

[5] E. Goursat, A Course in Mathematical Analysis, vol. 3, Part 2: Integral equations, Calculus of variations, Ginn \& Co., Boston, Mass. (1964).

[6] L.V. Kantorovich and V.I. Krylov Approximate Methods of Higher Analysis, Interscience Publishers, New York (1964).

[7] V. N. Kublanovskaya, Application of analytic continuation by change of variables in numerical analysis, Proc. Mathem. Inst. Acad. Sci. USSR, 53 (1959), pp. 145-185.

[8] S.E. Mikhailov, On an integral equation of some boundary value problems for harmonic functions in plane multiply connected domains with nonregular boundary, Matematicheskii Sbornik 121 (1983), pp. 533-544, (Engl. Translation: Mathematics of the USSR. - Sbornik. 1984; Vol. 49:525-536.).

[9] - Finite-dimensional perturbations of linear operators and some applications to boundary integral equations, Engineering Analysis with Boundary Elements 23 (1999), pp. 805-813.

[10] - Localized boundary-domain integral formulations for problems with variable coefficients, Engineering Analysis with Boundary Elements 26 (2002), pp. 681-690.

[11] - Localized direct boundary-domain integro-differential formulations for scalar nonlinear boundary-value problems with variable coefficients, Journal of Engineering Mathematics 51 (2005), pp. 283-302.

[12] - Analysis of united boundary-domain integro-differential and integral equations for a mixed BVP with variable coefficient, Mathematical Methods in Applied Sciences 29 (2006), pp. 715-739.

[13] S.E. Mikhailov and N.A. Mohamed, Iterative solution of boundary-domain integral equation for BVP with variable coefficient, in Proceedings of the 8th UK Conference on Boundary Integral Methods, D. Lesnic, ed., Leeds University Press, Leeds, UK, 2011, pp. 127-134.

[14] S.E. Mikhailov and I.S. Nakhova, Mesh-based numerical implementation of the localized boundarydomain integral equation method to a variable-coefficient Neumann problem, Journal of Engineering Mathematics 51 (2005), pp. 251-259.

[15] S.G. Mikhlin, Integral Equations and Application to Certain Problems in Mechanics., Pergamon Press, New York (1957).

[16] O. Steinbach and W. Wendland, On C. Neumann's method for second-order elliptic systems in domains with non-smooth boundaries, Journal of Mathematical Analysis and Applications 262 (2001), pp. $733-748$. 